\title{
Missing Energy Induced by Thin Hadron Calorimetry
}

\author{
Dan Green \\ Fermi National Accelerator Laboratory \\ P.O. Box 500, Batavia, Illinois 60510
}

April 1994 


\section{Disclaimer}

This report was prepared as an account of work sponsored by an agency of the United States Government. Neither the United States Government nor any agency thereof, nor any of their employees, makes any warranty, express or implied, or assumes any legal liability or responsibility for the accuracy, completeness, or usefulness of any information, apparatus, product, or process disclosed, or represents that its use would not infringe privately owned rights. Reference herein to any specific commercial product, process, or service by trade name, trademark, manufacturer, or otherwise, does not necessarily constitute or imply its endorsement, recommendation, or favoring by the United States Government or any agency thereof. The views and opinions of authors expressed herein do not necessarily state or reflect those of the United States Government or any agency thereof. 
Missing Energy Induced by

Thin Hadron Calorimetry

Dan Green

Fermilab

\subsection{Introduction:}

It is relatively straightforward to estimate the total, unsegmented, depth required in SSC experiments [1]. Typically depths in the range of 9-11 absorption lengths were specified by the SDC and GEM experiments. With these depths, the induced missing energy signal due to calorimeter leakage was found to be well below the signals caused by light gluinos [2], and the exterior muon systems were well protected from punch through backgrounds.

In certain applications, where calorimetry is inscribed inside the magnetic field producing coils, that depth of calorimetry becomes exceedingly expensive. Examples are the ZEUS barrel and the proposed CMS barrel calorimeters [3]. The problems can be alleviated by placing a "tailcatcher" of active elements outside the coil, but the problem of inert material placed within the active volume of the calorimetry remains. This inert material can potentially induce missing transverse energy signals which are large with respect to real physics signatures. The purpose of this note is to explore schemes to minimize the measured leakage energy.

\subsection{Truncation:}

A simple truncation is unacceptable at the high energies attained by the LHC and SSC. This assertion is illustrated in Fig.1 which shows "Hanging File" [4] data for $270 \mathrm{GeV}$ incident pions for a calorimeter of 9.22 nuclear absorption lengths (all depths will be quoted in these units in what follows) and a calorimeter truncated at a depth of 4.83 .

Clearly, Fig. 1 shows that $99 \%$ of the energies are measured within $+-20 \%$ of the true energy for the 9.22 deep calorimeter. For the truncated calorimeter, there is a shift in the mean of about $10 \%$, and $a \sim 3 \%$ tail where $>40 \%$ of the energy is lost. These effects are 
also energy dependent, so that an induced differential nonlinearity is a direct result of the truncation of the calorimeter depth.

Since a jet has a leading fragment with mean energy fraction, <z> $\sim 0.2$, a $1.35 \mathrm{TeV}$ jet will leak a missing energy of at least $\sim 100$ $\mathrm{GeV}$ at least $3 \%$ of the time. Given that the QCD jet cross section greatly exceeds the gluino cross section, the truncated calorimeter will compromise the Physics which is available with a good clean missing Et signal

\subsection{Passive Weighting:}

The effects of truncation can be mitigated by using longitudinal segmentation. The deeper segments should be weighted high, as they sample hadron showers which develop deep in the calorimeter, and hence cause large leakage. Such "exit weighting" has been extensively studied [4], and was planned for in the SDC experiment. As a rule of thumb, exit weighting is similar in effect to an unweighted calorimeter of depth $\sim 1$ longer.

Operationally, one may implement passive weighting by using optical filters or by adjusting electronic gains of individual sampling layers. If longitudinal compartments are read out separately, the weighting can be implemented as larger relative calibration terms for the deeper compartments.

The passive weights for a 5.9 deep compartment with the weight of the last 0.7 depth floating, followed by a 1.4 inert coil, and a "tailcatcher" 2.1 deep with a second floating weight were studied using test beam hadron calibration data from a neutrino detector [5]. Incident energies of 50,200, and $450 \mathrm{GeV}$ were used, and the "deep calorimeter" was $\sim 28$, or effectively infinite. The energies were defined to be, $E 1$ = energy in the first 5.2 depth, $E e=$ energy in the subsequent 0.7 segment (5.2-5.9 in depth), and $E t=$ energy in "tailcatcher" at depth 7.3-9.4 following an inert 1.4 deep "coil" simulated by failing to readout the $\mathrm{Fe}$ samples at those depths. Note that a real aluminum coil is more favorable than this model. The low $Z$ coil will not absorb the EM part of the shower as seriously as the inert Fe samples used in this model. Thus, this study serves as a worst case scenario.

The "exit" and "tailcatcher weights" were varied to find the condition of optimal weighting. The calorimeter energy was defined to be; 


$$
E=E_{1}+a_{1} E_{e}+a_{2} E_{\imath}
$$

The optimal weights were found to be $a_{1}=1.5$ and $a_{2}=2.0$. The contours of the fractional energy resolution as a function of $\left(a_{1}, a_{2}\right)$ are shown in Fig.2 for an energy of $450 \mathrm{GeV}$. Other energies were studied, and the optimal weight was found to be only weakly energy dependent. Therefore, one could passively weight the 2 compartments and use a single readout in depth with the optimal weight scheme. This single readout would be the cheapest scheme that could be implemented.

The resulting energy fractions are shown at 50,200, and $450 \mathrm{GeV}$ for the very deep calorimeter and the "CMS model" calorimeter with passive optimal weighting in Fig.3. Clearly, the rms of the CMS model calorimeter is somewhat broader than the very deep calorimeter. However, the tails are not evident. At $450 \mathrm{GeV}$ less than $1 \%$ of the energies are mismeasured by more than $20 \%$. The optimal weighting also restores the mean, so that the induced differential nonlinearity is quite small. Clearly, even this simple first step goes a long way towards mimicking the very deep calorimeter.

\subsection{Segmented Readout:}

It is natural to explore possible improvements beyond simple passive weighting of the deeper samples of the truncated or interrupted calorimeter. There is a clear correlation between the exit sample energy and the tailcatcher energy, as seen in Fig.4. One might expect that separate readout of the 3 compartments in the CMS model would yield improved energy resolution. In order to study individual readout, the CMS model calorimeter energy was defined to be;

$$
\begin{gathered}
E=E_{1}+\left[a_{1}+a_{2}\left(f_{e} / f_{1}\right)+a_{3}\left(f_{t} / f_{e}\right)\right] E_{e} \\
f_{1}=E_{1} /\left(E_{1}+E_{e}+E_{t}\right) \\
f_{e}=E_{e} /\left(E_{1}+E_{e}+E_{t}\right) \\
f_{t}=E_{t} /\left(E_{1}+E_{e}+E_{t}\right)
\end{gathered}
$$


The six parameters, $a_{i}$, were varied such as to minimize the sum of the squares of the differences between $E$ and the energy of the deep calorimeter on an event by event basis. The resulting distributions, analogous to Fig. 3 for optimal weighting, are shown in Fig.5a and Fig.5b for 450 and $50 \mathrm{GeV}$ respectively. In comparing to Fig.3, one can see that the residual tails which exist for optimal weighting are pulled in somewhat. However, the gains are not very large. This functional form may not be optimal. The basic idea was to use the energy fractions in the 3 compartments, $f_{1} f_{e}$ and $f_{\mathrm{t}}$ as indicators of the shower development of the individual fluctuating events.

A direct comparison of optimal passive weights and fitted weights is shown in Fig. $5 \mathrm{c}$ for $450 \mathrm{GeV}$ data. The fractional deviation from the energy of the deep calorimeter, on an event by event basis, is plotted for both cases. Clearly, the energy is contained at the $1 \%$ of events level to $+-15 \%$ for fitted events compared to +- $25 \%$ for optimally weighted events.

\subsection{Summary, Conclusions:}

The requirement of $a \sim 10$ deep calorimeter can be evaded at the cost of longitudinal segmentation and increased number of readout channels. For energies $<450 \mathrm{GeV}$ it appears that only slightly degraded Gaussian errors can be attained by weighting a 5.9 deep calorimeter interrupted by a 1.4 deep "coil" and augmented by a 2.1 deep "tailcatcher". The Gaussian errors are known to have minimal effect on multijet spectroscopy $[6,7]$. Since the non-Gaussian tails due to truncation are evaded using several compartments with separate readout, the missing Et Physics (neutrino signals) is also not compromised. Therefore, thin hadronic calorimetry is not necessarily such to compromise the Physics if sufficient care is taken in the construction and readout of the device. 


\section{References}

1. D.Green et al., Depth Requirements in SSC Calorimeters, SDC19-00016, Fermilab FN-570 August, 1991

2. Solenoid Detector Collaboration, Technical Design Report, SCD92-201, April 1, 1992

3. CMS, The Compact Muon Solenoid, Letter of Intent, CERN/LHCC 92-3, October 1, 1992

4. A. Beretvas et al., "Beam Tests of Composite Calorimeter Configurations from Reconfigurable - Stack Calorimeter", Nuc. Inst. and Meth.A329, 50 (1993)

5. Andy Beretvas, private communication.

6. A. Para et al., "Jet Energy Resolution of the SCD Detector", SDC90-149

7. D. Green, "Dijet Spectroscopy at High Luminosity", SDC 90-070

\section{Figure Captions}

Figure 1. Hanging file data taken with $270 \mathrm{GeV}$ incident pions. The distribution of energy is plotted for a $4.83, \cdots-$, and a 9.22, - . *, interaction length calorimeter. The shift in the mean and the long tail at low energy exhibited by the truncated calorimeter is quite evident.

Figure 2. Data taken with $450 \mathrm{GeV}$ pions incident on a very deep calorimeter. The contour of the fractional energy is plotted as a function of the constant weight assigned to the exit portion and the tailcatcher portion of the $5.2+0.7+1.4+2.1$ absorption length segmented calorimeter. 
Figure 3. Distribution of energy/beam energy for a $5.2+0.7+1.4+$ 2.1 segmented calorimeter with optimal weighting of the 0.7 exit compartment and the 2.1 tailcatcher compartment ,- - - - *, compared to a very deep calorimeter, ---- o, for $450, a, 200, b$, and $50, \mathrm{c}, \mathrm{GeV}$ incident pion energy.

Figure 4. Scatter plot of unweighted exit energy, in $\mathrm{GeV}$, vs tailcatcher energy, in $\mathrm{GeV}$, for $450 \mathrm{GeV}$ pions incident on a $5.2+0.7+1.4+2.1$ segmented calorimeter.

Figure 5.a, 5.b The distribution of calorimeter energy/beam energy for a $5.2+0.7+1.4+2.1$ segmented calorimeter with fitted weights, - . * , incident pions respectively.

Figure 5.c

Fractional energy error of optimally weighted, - - 0 , and fitted weights, .... *, for a $5.2+0.7+1.4+2.1$ segmented calorimeter with respect to the full depth calorimeter compared event by event. 


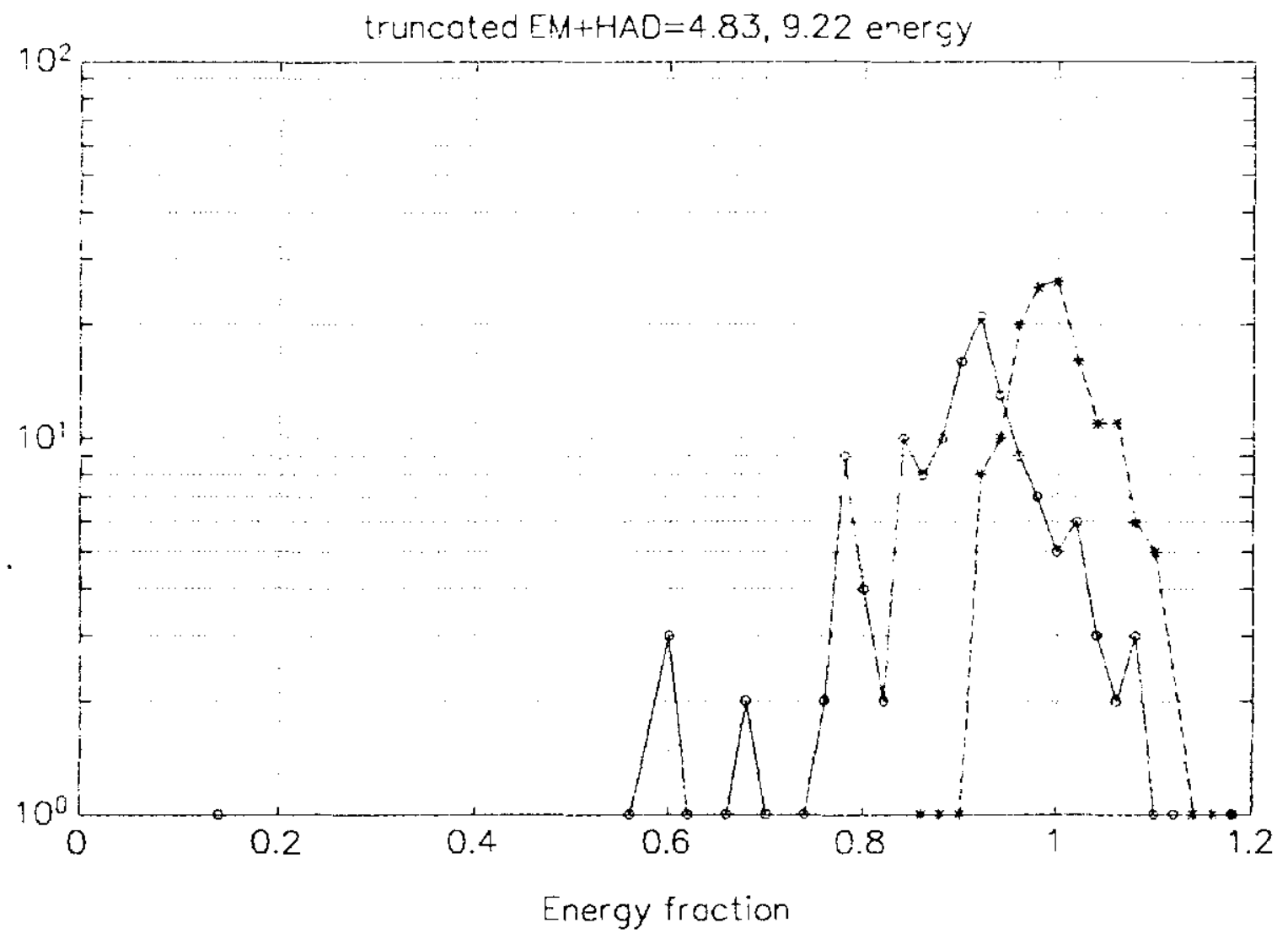

Figure 1 
exit wt and tail wt dependence of $\mathrm{dE} / \mathrm{E}, 450 \mathrm{GeV}$

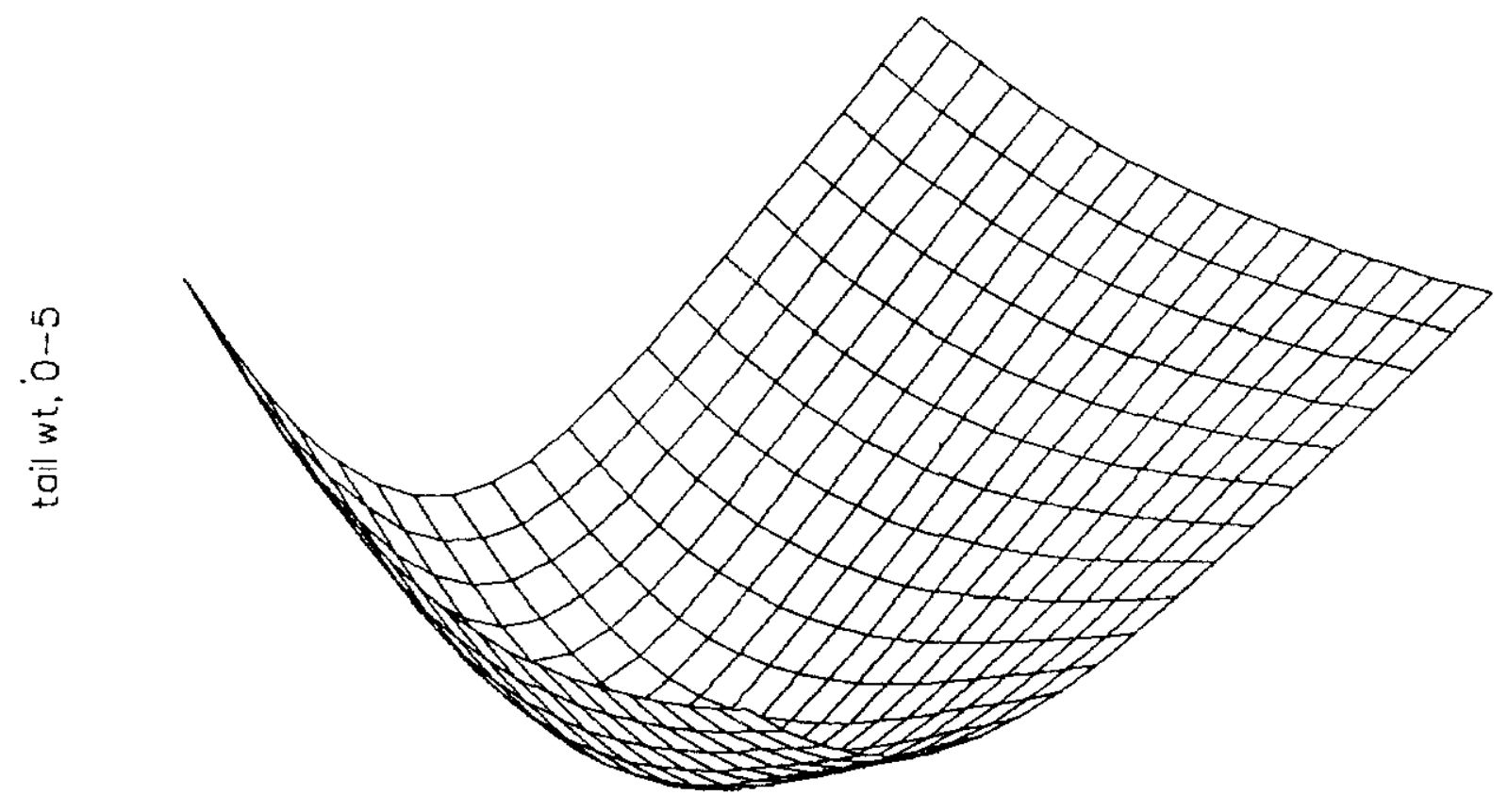

exit wt, 0-5

Figure 2 


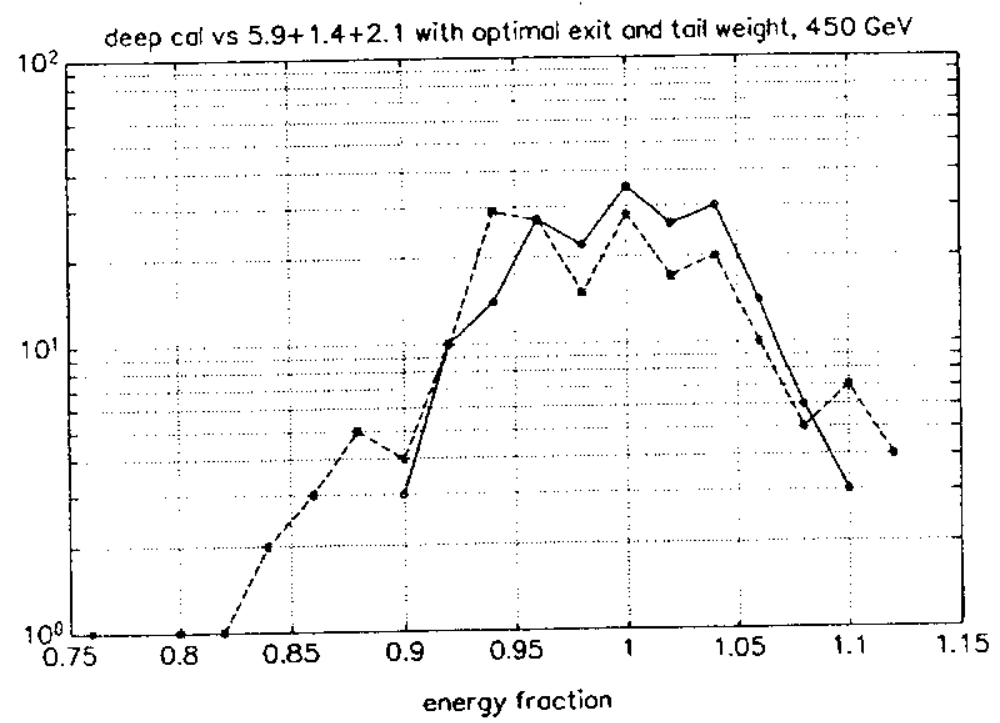

Figure 3 a

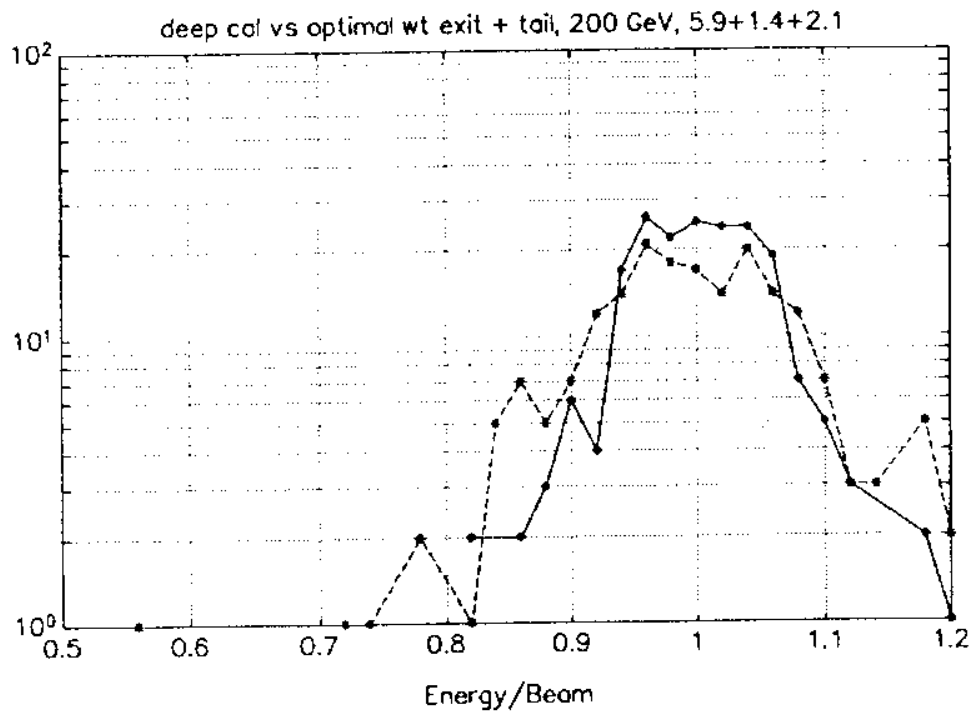

Figure $3 \mathrm{~b}$

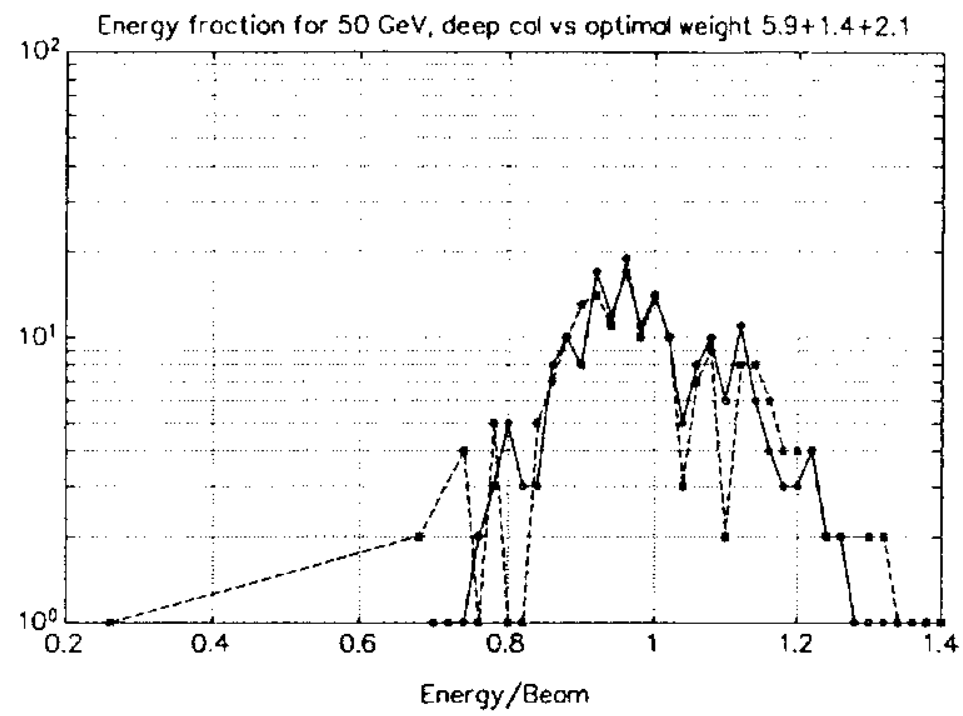

Figure $3 \mathrm{c}$ 


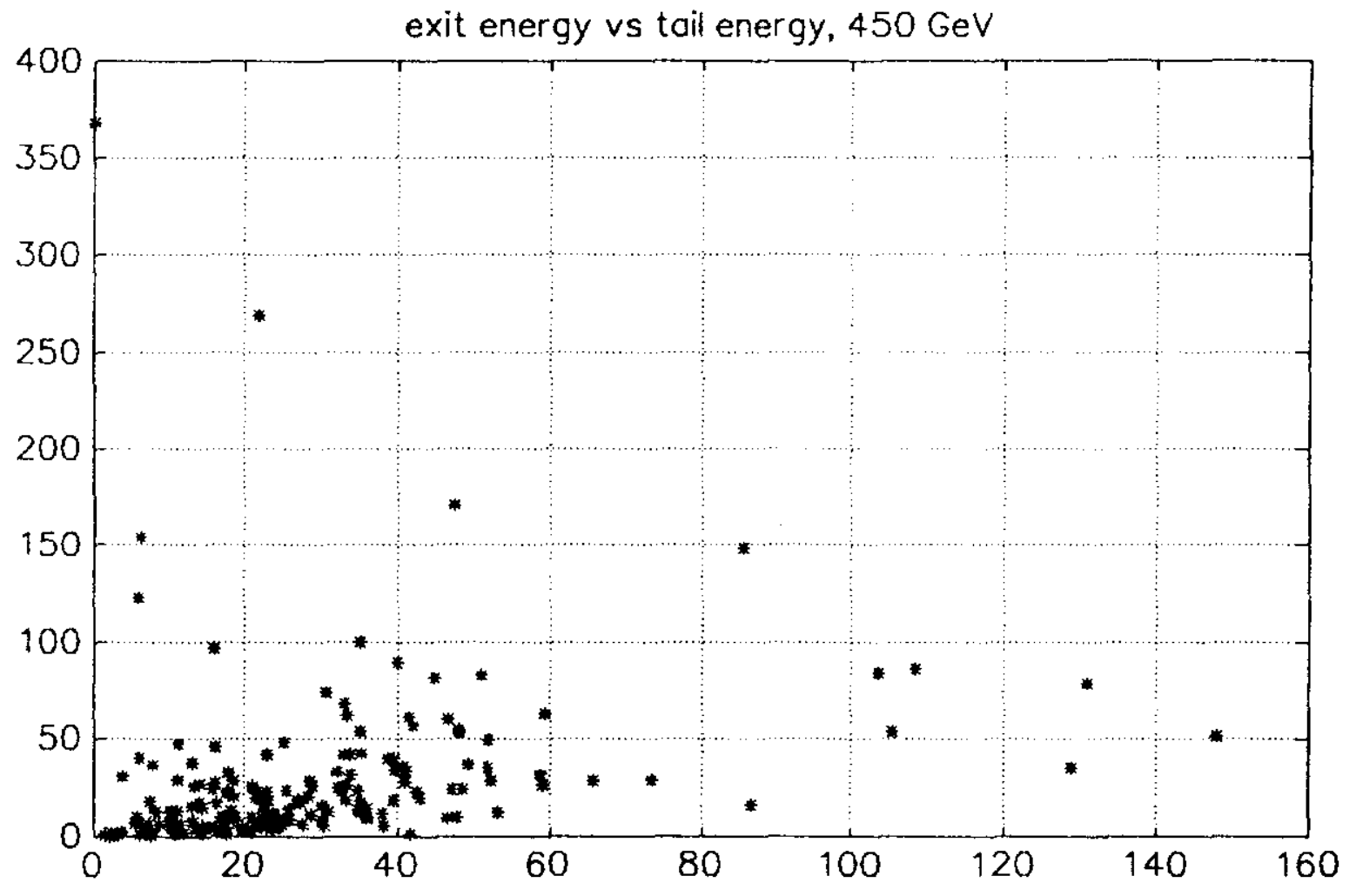

Figure 4 


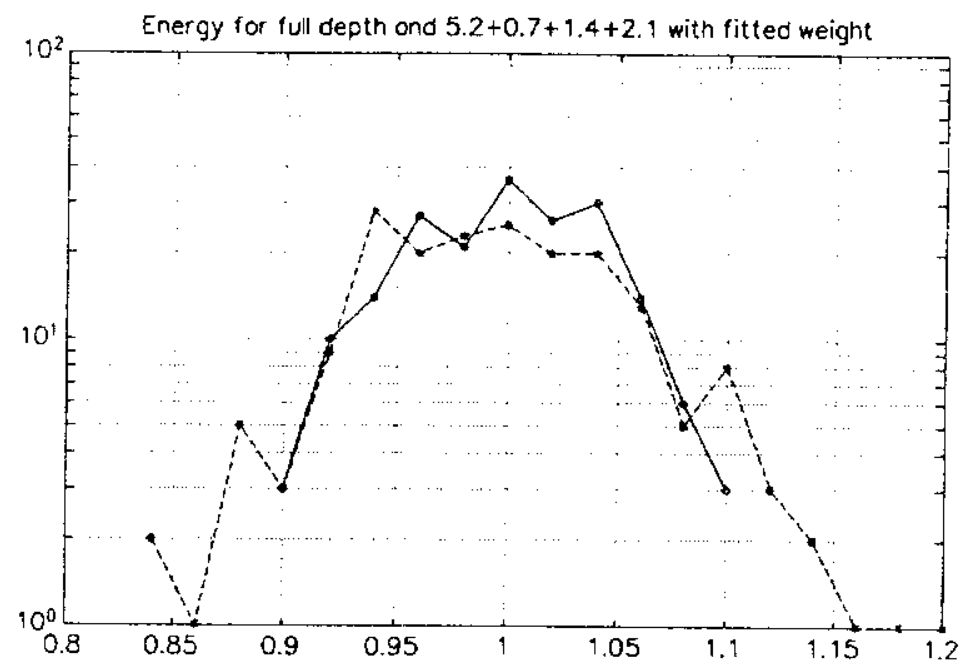

Figure 5 a

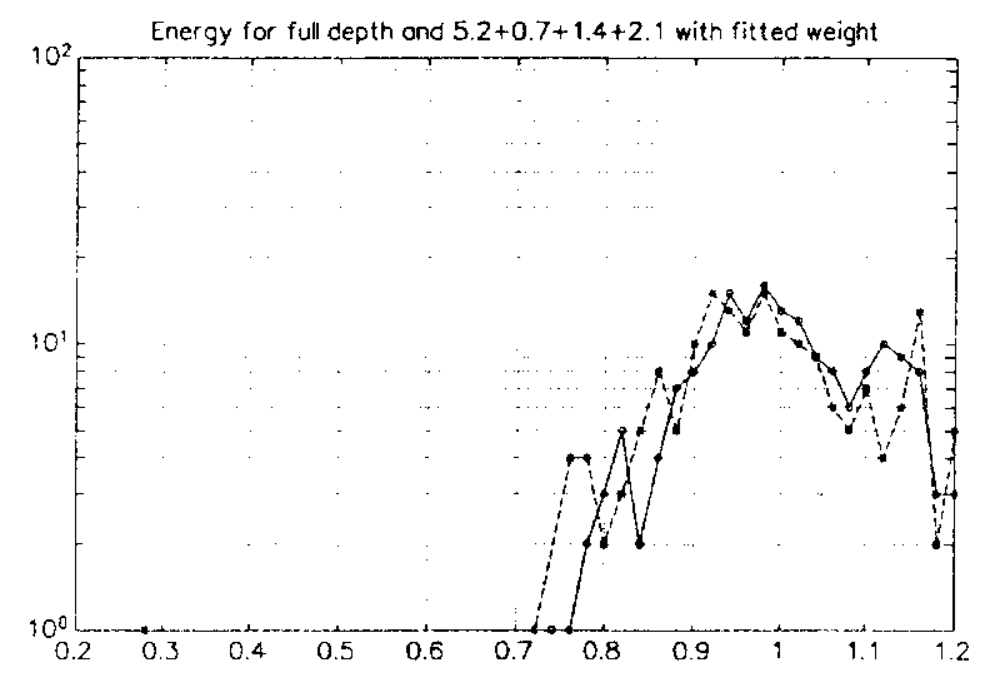

Figure 5 b

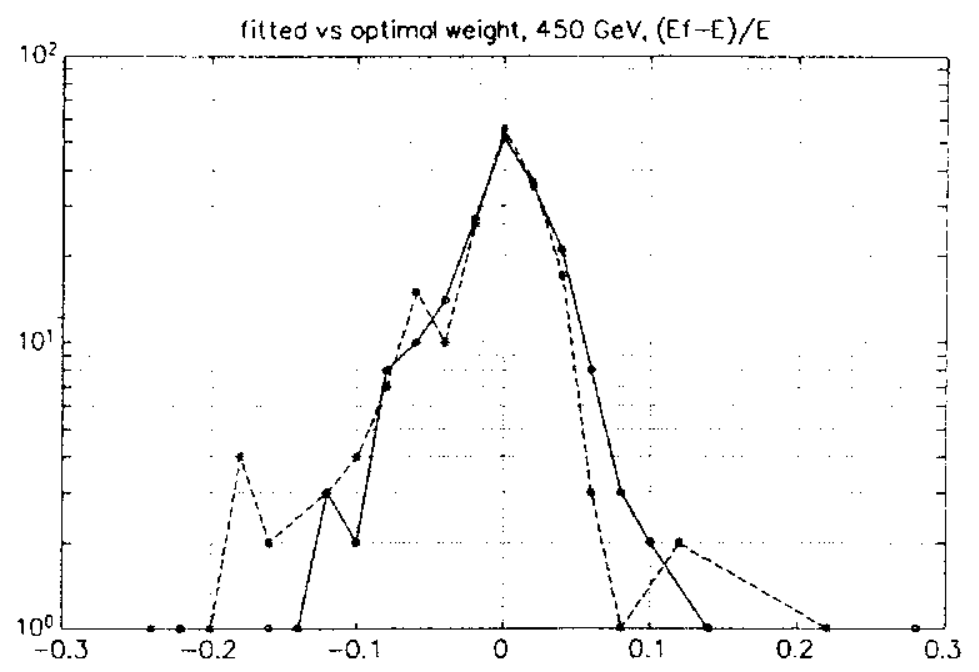

Figure $5 \mathrm{c}$ 